\title{
Analyzing the Internet Filtering Policies in KSA and USA
}

\author{
Zohair Malki \\ Department of Information and Learning Resources, The collage of Computer Science and Engineering, Taibah University, Yanbu Al Bahar, \\ Al Madinah Province, Saudi Arabia
}

Email address:

zmalki22@gmail.com

\section{To cite this article:}

Zohair Malki. Analyzing the Internet Filtering Policies in KSA and USA. International Journal of Science, Technology and Society. Vol. 3, No. 3, 2015, pp. 83-89. doi: 10.11648/j.ijsts.20150303.13

\begin{abstract}
This paper attempts to define internet filtering and outline the purpose of enforcing the filtering policy, and analyze its effect on the use of the Internet resources. This study further examines and evaluates the regulations regarding Internet access in Saudi Arabia, with an emphasis on the problems that people come across during Internet access with limited access to specific web pages due to the policy of filtering. This paper will also examine the case of the Supreme Court of the United States appellant vs. American library association which is similar to that of the Saudi Arabian case with miniscule differences only in term of ritual and cultural aspects. The paper evaluates the advantages and disadvantages of using the filtering policy and its influence and application to the culture and religion of Saudi Arabia. The study further enlists some of the rules and regulation, developed about this issue with the reference of the case of the Court of the United States appellant's vs. American library association. Finally, this study discusses the impact of different cultural and tradition on the ways of application of the filtering strategy in both sides.
\end{abstract}

Keywords: Filtering Policy, KSA Internet Filtering, American Library, American Library Association

\section{Introduction}

In the present day, the World Wide Web has transformed the way of communication, now the entire world seems to be a small village with an ease of access of information than ever before. The recent decade has witnessed a revolution in the popularity and volume of the Internet. Today the Internet is serving millions across the globe as a hub of information and connectivity through remote access. It is also connecting people to information, electronic mail, and tools of delivery for information products. A variety of information that was almost incomprehensible earlier has been made extensively and effortlessly accessible through the web. (Zittrain \& Edelman, 2002) However, most of the users of the Internet services are unaware of the rules and regulation of using these services.

Policies affecting the flow of Information and underlining the consequences of the use of technology and accessing available resources through the Internet or databases have received a lot of emphasis in the field of information studies. In the present day while the Internet has become a massive information resource, it must be used wisely and selectively as some of its content is found to be inappropriate for the users and also has experience of dangers of its use in the past.
For example, obtaining illegal or private information on certain people or countries is amongst a commonly practiced vice on the internet. Information Technology is a vital concept for the modern world and is defined as the skill of the new generation. Also, it is imperative to understand the acceptable and unacceptable usage of this new technology in the country as per its traditions and value systems and how the policy related to these services, sometimes protects the users from being affected by illegal practices of its use and at the same time prevents the users from accessing to a particular type of information. It is also essential to consider the access needs of the users and the effect of the rules and regulations on its usage. (Banks, 1998).

In the Gulf countries especially Saudi Arabia, one of the difficult decisions was allowing the access of the Internet to the public, because of its conservative and religious ways of governance. Hence, it took two years by the government to approve Internet access to the public, that too after applying very restrictive policy. As from the illegal and undesirable use and the copyright laws, the access to the Internet services needed to have policies, which were compatible with the rules of the country and consistent with the user's belief. The policy states that citizens should have the right to use the services in an atmosphere that is conducive to the successful 
completion of Internet services. The Saudi government installed filtering systems to prevent the public access to certain web pages and sites. The question that needs to be asked now is how does this policy come together? It seems that different cultures tend to develop different values, self-concepts, beliefs, lifestyles, and ways of providing services (Burger, 1993). For this reason, there was an obvious need for more comprehensive research to explores and analyzes the problems and the influence of the application of such filtering policy on the culture and tradition of Saudi Arabia.

Allowing Intellectual freedom in the information hungry society with filtering software's is abroad topic requiring deliberations evaluation and analysis. As it can be seen, there are many people who may agree with this concept; however others may not. Although it is now a popular and widespread practice in many places such as companies, libraries, universities, schools, and other providers of Internet access, it remains a controversial topic amongst a section of the population. (Busha, 1972). This paper will explain internet filtering, outline the purpose of applying the filtering policy, and elucidate the filtering policy and its effect on the use of the Internet resources. This paper attempts to investigate and analyze the Internet access policy in Saudi Arabia. It will specifically explore the problems encountered by the people while accessing the Internet with the filtering policy to restrict the access to specific web pages.. The paper also throws light on the advantages and disadvantages of applying this policy and the influence of the culture and religion to the obligation of applying this policy and vice versa. This is followed by the case of Supreme Court of the United States appellant's vs. American library association and some of the rules and regulations which have been developed about this issue. This is quite similar to the Saudi Arabian case where the only differences are in terms of culture and tradition aspects. Lastly, the paper also discusses the details about the effect of the different cultural and tradition on the application of the filtering policy.

The term filtering may be defined as - "Filtering is the process by which particular source or destination addresses can be prevented from crossing a bridge or router onto another portion of the network" (Network Buyer's, 1999). or "The use of a program to screen and exclude from access or availability the webpages or e-mail that is deemed objectionable" (Techtarget, 2003).

Content filtering is widely being used by corporations as part of Internet firewall computers and also by owners of personal computers in their homes, especially by parents to screen the inappropriate content their children have an access from a computer (Tech target, 2003).

\section{Internet Filtering in Saudi Arabia}

Since 1994, Saudi Arabia (KSA) has been connected with the Internet, but the use was restricted on the medical, state, academic and research purposes (Yam, 2002). The public in Saudi Arabia could subscribe to local intranet found inside the country such as Al-Naseej. In Al-Naseej network they can use public e-mails and link to local chat rooms. However public can't directly use World Wide Web (Al-Sarami, 1999). Starting Juanuary 1999 Saudi Arabian government starts to allow Saudi users to enter public internet services. Since then Saudi government started to strict the online contents available for internet users and applied filtering policy for public accessed online content. Officials of the Saudi government and the king Abdul-Aziz City for Science and Technology (KACST), the Riyadh-based state institution were made the pivots for coordinating the Internet policy. They targeted to exercise control over Internet content and make a decision about the appropriateness of the contents for the users. Moreover, the KACST was made responsible for issues, operation, regulations maintenance and policies governing the usage of the service, and also for the development of the Internet. (Al-Rasheed, 2001).

Figure 1 shows a list of a number of internet users in KSA year by year expressing the increasing amount of internet users in KSA.

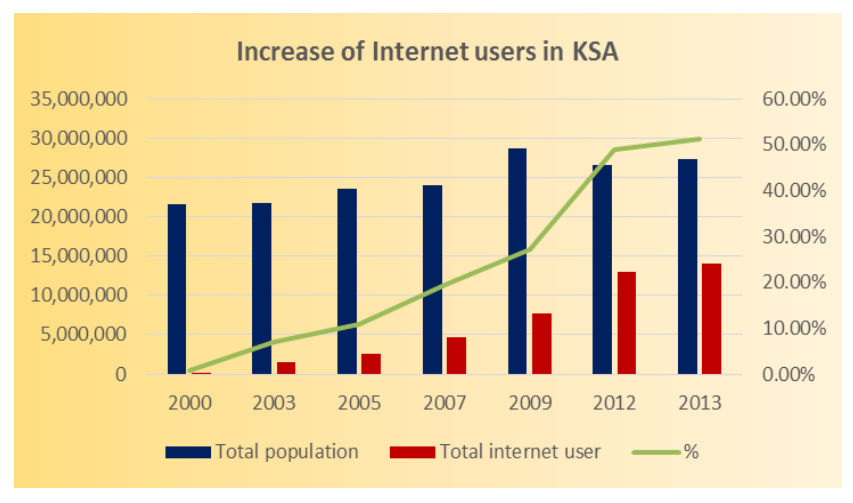

Figure 1. Express the increase of internet users in KSA.

A standing board of trustees framed and affirmed by the government secures the general public by avoiding such material on the Internet that abuses Islam or infringes on the conventions and society of the individuals of the nation (Al-rasheed, 2001). This committee classifies the inappropriate sites as immoral, such as pornographic, sites with sexual content, anti-Islam sites and other. Furthermore, it blocks the subscribers from entering such sites that have any such explicit materials or content.

The Internet is swarming with a lot of explicit an inappropriate content; hence, the government has launched a system to prevent such content from influencing the society. Such system ensures that users subscribed to this service will not receive any inappropriate content in their computer (Yam, 2002). The KACST officials are equipped with software, procedures, and hardware that inhibit internet user from accessing any online content that degrades, or that harms the Islamic Religion values and Muslims traditions. They also installed "firewall" or internet barrier that prevent other parties infringe their websites. This is the reason the government of Saudi Arabia didn't try to quickly provide the service for public, and it is inclined to remove all undesired online contents from being accessed by internet users 
(KACST, 1998).

Saudi Arabia's Council of Ministers endorsed this objective early on and called for firewall, administrated by KASCT and prevented users from accessing improper online content. In addition The Council of Minsters created set of rules and policies that control the internet accessing and usage (Zittran \& Edelman, 2002). In May 1998, the decision was made public, and it restricted the Internet Services Provider( ISPs) and internet users to surf the internet for prohibited acts such as online gambling, pornography or doing illegitimate activities that violates the social economics, culture, politics and religion. (Al-Adhel, 1998).

(Figure 2 shows a diagram that expresses the niches and blocked websites by KSA. It is not about the pornography websites only but also for various niches.)

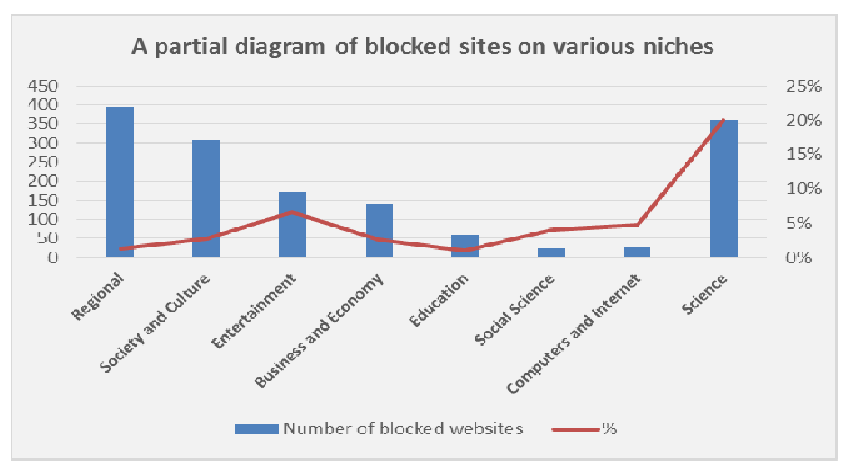

Figure 2. A partial diagram of blocked sites on various niches.

The list is provided by one of the giant search engines named as "Yahoo." Although, this is not the exclusive evidence but still it expresses the picture of the KSA partially.

There is a common thinking that KSA only blocks those sites that contain sexual contents only. But it is not like that. The filtering system of KSA blocks different types of sites on various niches. Even some religious pages are blocked in KSA. Yahoo published list of various websites from different niches in the year of 2012 which are blocked in KSA. Table 1 shows some exclusive part of that list.

Table 1. Examples of few blocked sites on various niches.

\begin{tabular}{|c|c|c|}
\hline $\begin{array}{l}\text { Niche/theme of the } \\
\text { website }\end{array}$ & $\begin{array}{l}\text { Example from } \\
\text { blocked sites }\end{array}$ & $\begin{array}{l}\text { Number of total } \\
\text { blocked sites }\end{array}$ \\
\hline Religion & al-bushra.org & 246 \\
\hline Education and reference & women eb.com & 45 \\
\hline Humor sites & createafart.com & 81 \\
\hline $\begin{array}{l}\text { Entertainment, music, } \\
\text { and movies }\end{array}$ & foxsearchlight.com & 251 \\
\hline $\begin{array}{l}\text { Pages about Middle } \\
\text { Eastern politics, } \\
\text { organizations or groups. }\end{array}$ & hizbollah.org & 80 \\
\hline $\begin{array}{l}\text { Services allowing } \\
\text { circumvention of } \\
\text { filtering restrictions }\end{array}$ & systransoft.com & 250 \\
\hline Pomography & Elephanttupe.com & 1300 \\
\hline
\end{tabular}

Sometimes internet filter of KSA blocks few sites temporary. Their previous records said that they blocked some famous websites for various reasons at different time. Google and Yahoo published a list jointly in June, 2002 and Table 2 shows few taglines from that list.

Table 2. Example of few temporary blocking sites by internet filter in KSA.

\begin{tabular}{ll}
\hline Name of the Website & Blocking Dates \\
\hline \multirow{2}{*}{ www.amnesty.org/ailib/intcam/saudi } & May 18, May 19, May 22, May \\
& 24, May 27 \\
www.1-marijuana-seeds.com & May 19, May 22, May 24, May 27 \\
www.al-bushra.org & May 16, May 18, May 19, May \\
& 22 , May 24, May 27 \\
world.altavista.com & May 18, May 19, May 22, May \\
& 24, May 27 \\
www.angelfire.com/ca2/queermuslim & May 18, May 19, May 22, May \\
& 24, May 27 \\
www.answering-islam.org & May 18, May 19, May 22, May \\
& 24, May 27 \\
www.bahai.com & May 14, May 18, May 19, May \\
& 22, May 24, May 27 \\
\hline
\end{tabular}

Although as per the official version of filtering internet content primarily focuses on online materials which are considered offensive to conservative Muslims. In Saudi Arabia the restriction and filtering phenomenon also applied to sites with political content. As an example, in the early 1999, the Committee against Corruption website in Saudi Arabia that is an exiled dissident group faced unauthorized blocking. Users in Saudi Arabia trying to surf forbidden sites get blocked with onscreen message that tells them that the website is forbidden, as shown in Figure 3. The logging into forbidden sites is reported and recorded. Saudi government has left no stone unturned to prevent any effort by the users to evade censorship. Recently, the URL address of common websites for online proxy server facilitating anti-censorship and a web site offering anonymous services was blocked in the Saudi Kingdom. (Zittran \& Edelman, 2002).

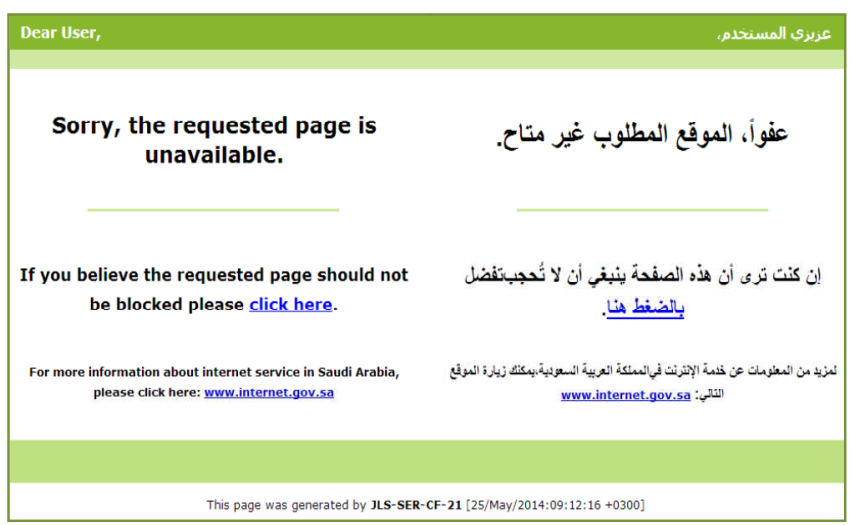

Figure 3. The warning message displayed when trying to access violated sites in Saudi Arabia.

\subsection{The Disadvantage of Filtering}

The filtering policy imposed by the Government has posed pair of threatening points to the right of publishing information and freedom of expression.

- $\quad$ Firstly it applies prior censorship to the internet content, 
so, each content has to be subjected to a hard level of blocking.

- Secondly, the filtering technologies developed till date is imprecise tools for content filtering in practical terms.

Even though the motivations for preventing online surfing such as keeping adult content and material away from users have been justifiable, the tools for filtration have intentionally or else, spontaneously blocked the legal surf of cultural, political, and medical content which are available and legal internationally with international standards and laws.

KACST under government orders blocks the selected websites based on their content. This blocking also extends to inappropriate cultural or political materials and content (Yam, 2002). A proxy server like the one used in KACST is also used by authorities to keep an eye the computer terminals accessing web sites and also the duration of such accesses. (Douglas1999).

The concept of filtering the Internet by the government has been successfully implemented in K.S.A despite of the fact that it may seem objectionable to some. Moreover, this policy gives the opportunity of request to the users, for instance any of the users of the internet service can complain request for unblocking using an unblock request form, in case a genuine website is intentionally blocked, stating the cause of why not blocking such site. Similarly, online users can send information about a website that they feel it is offensive and giving reasons for its blocking using a proxy report email address (Al-kdahi, 2003).

\subsection{The Filtering Mechanism in Saudi Arabia}

Various sources in the computer industry state that the K.S.A. System works in two stages. First, it approves web pages and stores them in a 500-gigabyte storage system in the capital city of Riyadh. Users access these pages from this computer system rather than the on the web. These are the frequently used pages, and they can be accessed quickly without the system having to assess their suitability each time. Requests received from the users to access the pages that are not stored in the cache, are scrutinized for suitability by the second stage of the system supplied by Websense, which is a U.S. based company, which lists and filters thirty categories of potentially unsuitable sites (Whitaker, 2000).

The policy of Internet filtering enables the government to:

- Prevent the public from accessing the online content not respecting Islam traditions or infringes on the people culture in the country

- Blocks any content interfering with the fundamental concepts for example; pornographic, sexual content, anti-Islam sites and other.

- Control on Internet activity or forbidding the distributing or online surfing of content that contains sensitive and valuable information for example, any news damaging anything negating a basic guideline or enactment, or encroaching the sacredness of Islam and its benevolent Shari'ah, or breaking open tolerability.

- Full control over the access to the Internet the ability to control over the service's providers and the connection to the sources of information.

\subsection{Some Practical Implications}

It is also a ground reality that the filtering software has been an imprecise tool, and it always blocks loads of material beyond its stated purpose. The most sophisticated products have similar issues even though they are checked by staff members on a regular basis for suitability of the websites. The filtering system becomes disadvantageous in term of blocking a lot of important information especially in the field of Education, health, religion, and entertainment. Users remain dissatisfied about these aspects of the filtering policy.

The Users satisfaction about KASCT (Open Net Initiative 2004) is shown in Figure 4. The filtering system in KSA has continually blocked the websites containing information on education, social, psychology, and health which has hampered the spread of knowledge about latest threats in these fields. For example, the sites having content about AIDS prevention is blocked because it has the words sex, woman, and intercourses in the content of the website. Even the website for pro-filtering was blocked because it shared a commercial server with a pornography site (Richtel, 1999).

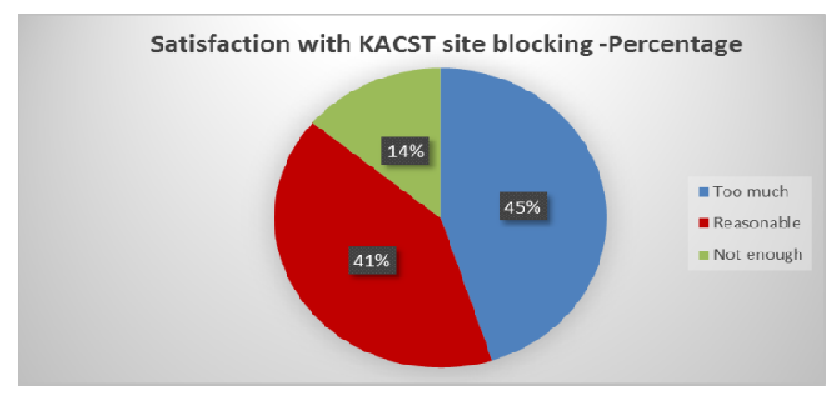

Figure 4. Users Satisfaction with KACST site blocking.

\section{Children's Internet Protection Act (CIPA): A Case from the USA}

The library associations and website publishers challenged constitutionality of children's Internet protection Act (CIPA). Under this proposal, any school or library where software for pornography filter was not installed would be prohibited from federal fund provided with the intention to help in the purchase of link to access the internet.

According to a letter signed by the Computer and Communications Industry Association and Information Technology Association of America, groups that belonging to the advanced technology productions, filtering obligation has become an obstacle for federal regulation of using the internet and accessing its content (CDT, 2000). In addition, Teacher associations and American Library Association have an objection on the blocking and filtering policies (Bruening \& Davidson, 2003). The objection of hard filtering policy said that mandatory filtering reflects a political viewpoint. They said that sometimes the filtering is flawed and they fail to block pornography.

Internet filtering including focus on the family is very 
important with respect to some conservative groups, and as per the American Family Association. "Children do not have a constitutional right to access and view Internet pornography in our local libraries (AFA, 2003)."

Although the court didn't apply forcing policy on content filtering, in part, The Supreme Court found that the filtering tools is less restrictive to protect users from ethically harmful and undesired content effecting their well-being; it has stated CDA to be unconstitutional. However, despite that COPA is likely vanish as a valid content law, there is still a burning issue among parents, legislators and conservatives which is the explicit sexually content and other hostile content (ALA, 2003). Last year, Congress issued a collection of national legislative proposals in response to that parental concern; it comes after making good survey for the media service by children and online safety. Most of the proposed legislation mentioned the requirement to install blocking and filtering technologies in universities, schools and libraries in which computers are gained by public funds (ALA, 1999)

Moreover, many teachers, scientists and Liberians said that it is very undesirable that some of the considered legislations always connect the use of the internet filtering policies with financial purposes. All education centers and schools, who get "E-rate" fund, are obliged to install filtering and blocking tools on their computers (ALA, 2002). The American Library Association, teachers, educators and local Liberians opposed such mandatory governmental filtering policies, alleged that they must be free to guide their students and children to the proper content and guide them to avoid improper content.

Moreover, many believe that it is very important for society and educators to discuss the role of then new inventions and technologies in the overall educational and public trends (ALA, 2003). It is considered constructive act to discuss such important ideas and exchange them, it will also encourage users to get benefits out of the electronic resources.

Many Libraries and schools have responded to the filtering controversy and the possibility of government regulation in many ways, schools and educators which using commercial filtering tools said that it is very restrictive filtering policies and prohibit in many cases the usual use of internet content.

In addition, in the "Filtering on Home PCs" part, many of the software tools are including some political and cultural trends and bias, that stop the access of internet content that the teacher, in some cases, need his students to get access to such content and evaluate it. As a result the commercial filters became not only filtering "Objectionable" sites but also became an obstacle in the education process. (Langland, 1997)

According to a study by the National Commission conducted in 1998 on Libraries and Information Technology policy, almost all libraries were found to have policies that govern Internet usage. However, only about $15 \%$ of these public libraries use filters.

Amendment 1501 passed by the House of Representatives was related to the Child Safety and Protection Act and it required that all libraries and schools have filters installed on their Internet access computers to block obscenity and child pornography. While the American Library Association (ALA) has no objection to the idea that all libraries install filters on their computers, the ALA believes that filters in libraries are not the only way to protect children and there are many places where children have threats due to access to the internet such as television, movie houses etc. The policy of ALA states that parents should supervise older and younger children when using the Internet and young children should never be left alone in the library.(ALA, 1999).

Conversely, ALA also raised concern in opposition to amendment 1501. The ALA believes this amendment will impose one size fits all federal mandates, which undermines the powers and local decisions made by libraries, schools, and their governing boards responsible to provide a safe and rewarding experience for children in using the Internet (ALA, 1999). It also states that the library users who access the Internet must be responsible for their own searches. Library has also set up guidelines to achieve this goal by offering various resource formats and services to meet the need of the community.

- These guidelines state that parents or guardians are responsible for supervising their children's Internet use and should convey to them the material they should not view and use.

- In addition, all citizens of the community can freely use the public library for Internet access, portioning to recreational purposes, which are limited by the Computer Courtesies list available in the library (ALA, 2003).

- According to library policy on Intellectual Freedom, mentions that anyone accessing sexual explicit or violent materials may lose their library privileges.

- Anyone found using the library for illegal and unacceptable use including harassment; slandering and violation of copyright laws will not only lose their library privileges, but may be subject to prosecution by local, state or federal authorities.

- The policy goes on to state that citizens should have the right to use the library in a conducive atmosphere for successful completion of library business (Langland, 1997).

\section{Analysis and Discussion}

It is clear that this internet filtering intends to protect the community as a whole and to maintain a certain moral standard throughout the country. On the other hand, the policies of the American government appear to be somewhat different from that of Saudi Arabia. These differences arise mainly from the well-known freedom of speech and knowledge in a democratic society. Intellectual freedom is a core value of the library profession and a basic right in a democratic society. In the United States, everybody is made responsible for their desire of knowledge, and it is deemed to be the government's obligation to provide the public with this freedom. Therefore, the application of the filtration of 
information over the Internet would surely contradict the core values. The rights of library users, to read, seek information, and speak freely are actively defended by the ALA as guaranteed by the First Amendment. It is quite clear that the ALA assists and promotes libraries that are helping children and adults develop the skills required to understand and effectively utilize information resources essential in the present day global information society. The ALA also affirms that the use of filtering software by libraries clearly violates the library bill of rights by blocking access to consistently protected speech (ALA, 2000). In this case, libraries and schools in the United States have the right to decide wither to apply filtering over the information on the Internet or not.

In USA most of the websites are open to all people. They have few laws and if any websites break those laws then that specific website will be blocked in USA. Here goes the list of laws which may be used to protect a specific group of people from entering few specific sites, the list is shown in Figure 5.

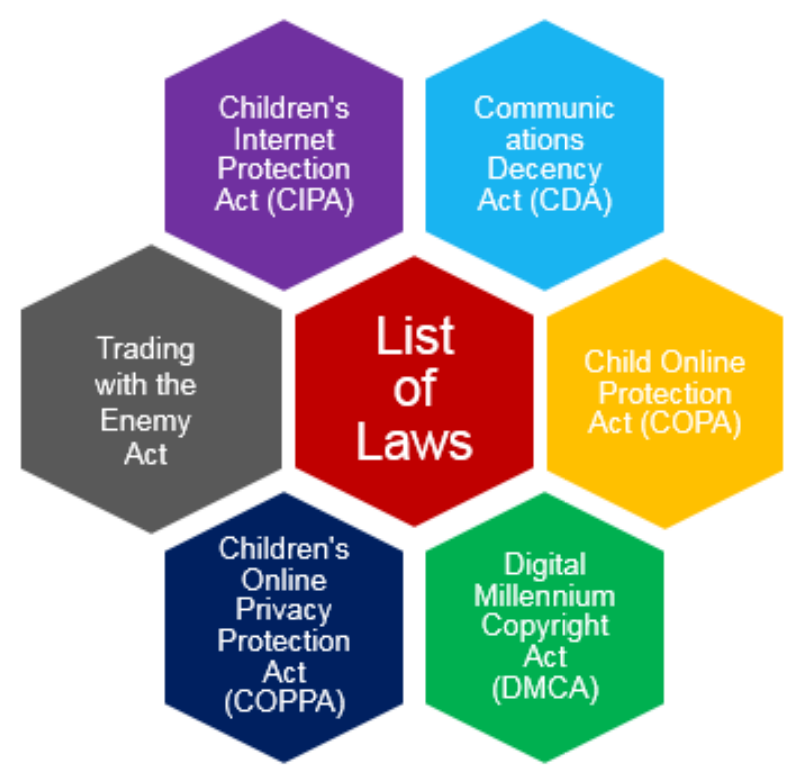

Figure 5. List of laws for preventing group of people of entering specific sites.

Besides these, almost $99.9 \%$ websites are open in USA but few of the government and private offices block few websites in offices at office hour so that employer can concentrate on office work perfectly. Figure 6 shows a list of those restricted websites with their percentage of restriction.

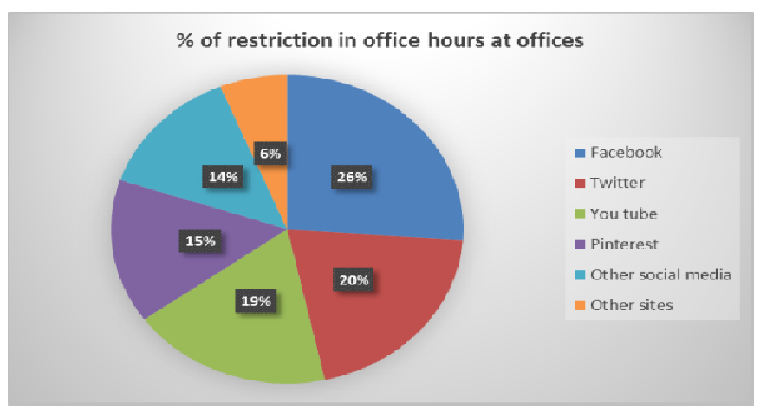

Figure 6. Percentage of restrictions in office hours.
In Saudi Arabia, on the other hand, libraries and schools do not have control over its own rules regarding Internet filtering. The Saudi Arabian government is responsible to manage the content of the web. Thus, the deciding on what type of filters is to be applied becomes a government enterprise ensuring cultural protection. In simple terms, the government like a censor investigates and scrutinizes the suitable content and makes it available to the users.

The handling of Internet filters by the government can be called a natural phenomenon. In the past, when the library being the only source of information in Saudi Arabia, information on politics, and sexual issues were kept in a separate special room of the library; which was studied by people only on special request. Because such kind of information is freely available over the Internet, the Saudi government has attempted to create the necessary filtration systems addressing all such matters (Al-Adhel 1998). In the Saudi Arabia, a standing committee approved by the government, has been formed to protect society from such material on the Internet that violates Islam or encroaches on traditions and culture of the people in the country. This committee including members from the Saudi public citizens will determine which sites are immoral, such as including pornographic, sexual content, anti-Islamic sites and others. (Al-rasheed, 2001)This strategic approach of the government of Saudi Arabia allows the involvement of its citizens to applying the policy of filtering. In term of freedom of expression, the Saudi governments has given its citizens the right to determine whether a piece of information should or should not be blocked because it contains useful information, or there is a need for these kinds of information, for instance research, education, and medical purposes.

This procedure starts with submitting request from the users, for instance, any user of the Internet service can complain in case the site is blocked, giving reasons on why it should be unblocked using an unblock request form. Similarly, users can submit information about any site they consider offensive and reasons explaining why it should be blocked using a proxy report email address (Al-kdahi, 2003).

Although the core questions arises that do young people have the right to explore information and entertainment resources online without divulging who they are and what are the rights of their parents? Library users also access the Internet, and they must be responsible for their own searches. Libraries also have set up guidelines to achieve this goal by offering various resource formats and services meeting the needs of the community. The parents or guardians are responsible to supervise their children during Internet use by explaining to them which materials they should not use or view.

In the United States, there is no restriction on adult to access any kinds of materials on the Internet. However, it may be noted that this freedom may be a source of trouble for those who intentionally use certain information to inflict moral, physical or financial pain to others. Identity theft is one such example where one uses the Internet posing to be someone else causing grave damages to those who are targeted. One 
must not completely rule out the issues related to filtration in the United States as there are several communities in US that would strongly support the idea of Internet filtration. While at the same time there are many who out rightly reject this matter.

In Saudi Arabia, restriction over the Internet materials applies for both children and adults. The Islamic rules form the basis of the Saudi Arabian culture. Hence in accordance to Islam, information such as pornography, anti-Islam sites, sexual content, and others are prohibited for both children and adults. This would explain the fact that no age exception by the Saudi government for the free, unfiltered use of internet.

\section{Conclusion}

Internet filters are an important tool to control and maintain the appropriate rating and scrutiny of Web content. However, it is suggested by the arguments on both sides that the application of filters varies across cultures. For instance, in the United States, the public influences the legislation related to such control mechanism. Although certain individuals consider that the application of Internet filters violates the free flow of information, while others argue that a control to a certain extent is needed to keep pornographic materials away from the underage audience.

Nonetheless, other related problems also arise when the cultural context dictates how filters are applied and/or implemented. A closer look at the Internet filters dilemma suggests that there is a censorship regardless of culture. How these filters are being applied is quite interesting. In other words, who are the policy makers? In the US, the Congress and major interest groups impose the policies and legislation regarding filtering lodge. With all fairness, regardless of the source of control, it is a form of censorship. In Saudi Arabia, the government being the only form of censorship is responsible to create and force Internet filtering policies to the society.

\section{References}

[1] U.S. V. American Library Association, 2013. Retrieved at October/24/2013.

[2] The American Library Association. (2013). Why is Intellectual Freedom. Retrieved on October/27/2013.

[3] The American Library Association. (2002). ALA applauds federal court ruling on the Children's Internet Protection Act. Retrieved on October/27/2013.

[4] The American Library Association. (2013). How Do You Guide Children When You Can't Be with Them 24 Hours A Day?. Retrieved on October/27/2013.

[5] [5] The American Library Association. (2013). What is Censorship, How Does Censorship. Retrieved on October/27/2013.

[6] The American Family Association. (2013). Your Children and the Internet. Retrieved on November/4/2013.
[7] Federal Trade Commission.( 1999). Children's Online Privacy Protection Rule--Comment P994504(American Library Association Submission). Retrieved on October/20/2013.

[8] Bruening, P\& Davidson, A. (2013). Constitutionality of Internet Filtering Mandate Challenged in Court. Retrieved on November/4/2013.

[9] The Center for Democracy and Technology. (2000). Free Speech. Retrieved at November/10/2013.

[10] Banks, M. (1998). Filtering the net library: the case ( mostly) in favor. Computer in libraries.18(3), 50.

[11] Zittrain, J \& Edelman, B. ( 2002). Documentation of the Internet Filtering in Saudi Arabia. Berkman center for Internet \& society Harvard School. Re-trieved on October/21/2013.

[12] Robert H. Burger. ( 1993). Information Policy: A framework for evaluations and policy research. Ablex Publishing. April, 1993.

[13] Busha, C. H. (1972.) Intellectual Freedom and censorship: The climate of opinion in midwestern public library. Library Quarterly, 24(3), 283-301.

[14] Yam, A. (2002) Saudi Arabia censors the web. It Matters. More then the Bits and Bytes. Retrieved on October/21/2003.

[15] Al-Sarami, N. ( 1999). Problems and Possibilities; Internet in the Kingdom. Saudi Gazette, March 13.

[16] Al-Rasheed, A. (2001). The Internet in Saudi Arabia ( management view). Communications engineering technical exchange meeting 2001, 30 April- 2may. ARAMCO, Al-dharan.

[17] Langland, L. ( 1997). Public libraries, Intellectual freedom, and the internet: To filter or not to filter. PNLA Quarterly 16 (3).14.

[18] Al-Adhel, A. (1998). The regulations on ISPs forbid them from establishing any linkage to the Internet except via the KACST. The rules were published in the May 6, 1998. Al-Jazira daily, as reported in FBIS.

[19] Network Buyer's.(1999). Glossary of Industry Terms. Retrieved on October/27/2003.

[20] TechTarget Corporate Web Site. (2003). Filtering Definition. the guide to the Tech Target network of industry-specific IT Web sites. Retrieved on Octo-ber/29/2003.

[21] Douglas, J. (1999). The Internet's 'Open Sesame' Is Answered Warily," New York Times. March 18.

[22] Al-Kdahi, M. (2003).King Abdulaziz City for Science \& Technology (KACST). The Internet Services Unit. Retrieved on November10/2013.

[23] Whitaker, B. (2000). Saudi claim victory in war for control of web. The Guardian, 11 May.

[24] Richtel, M. (1999). Tables Turn on a Filtering Site As It Is Temporarily Blocked. New York Times, March 11.

[25] The American Library Association. (1999). American Library Association and ACLU Court Challenge to Children's Internet Protection Act (CHIPA)1999. Retrieved on October/5/2013. 